\title{
The Role of Intrinsic Noise in Origin of Quorum Sensing
}

\author{
Sarangam Majumdar \\ Department of Mathematics, University of Hamburg, Germany
}

Copyright $(2016$ by authors, all rights reserved. Authors agree that this article remains permanently open access under the terms of the Creative Commons Attribution License 4.0 International License

\begin{abstract}
Many biological phenomena about quorum sensing in bacteria are driven by experimentally and mathematically in the last few decades. Symbiosis, virulence etc. are the major area in which these theoretical models work well. But no specific origin was established so far which can explain quorum sensing itself. This weakly nonlocal fluid dynamical model of bacterial communication system introduces a pancake model which involves the intrinsic noise of the system to explain the origin of quorum sensing. The internal structure of quorum sensing analogues to Zeldovich model and origin of internal noise in this mechanism is responsible for quorum sensing.
\end{abstract}

Keywords Quorum Sensing (QS), Quorum Sensing Molecule (QSM), Noise, Viscosity, Zeldovich Approximation

\section{Introduction}

The phrase "Quorum sensing” means a cell-cell communication that bacteria use to assess their population density in order to coordinate the gene expression of the community [3].QS mechanism has a group behavior and the multicellular activity. The bacterium Psedomonas aeruginosa, Vibrio fischeri, Vibrio harveri, Vibrio cholera and many more use this QS mechanism which was observed about 30 years ago. The behavioural traits vary dramatically with the population density [2]. Bacteria determine their population density by producing diffusible quorum sensing molecules (QSM) and switch on the behavioural when the population of bacteria increases so too does the QSM concentration, until some apparent threshold is reached. Quorum sensing is considered in the context of cell - cell signaling between intra and inter species. The communication process is coordinated by quorum sensing molecules (autoinducer). QSM have some characteristics feature (1) the production of the quorum sensing signal takes place during specific stages of growth, under certain physiological conditions, or in response to environmental changes; (2) the quorum sensing signal accumulates in the extracellular milieu and is recognized by a specific bacterial receptor; (3) the accumulation of a critical threshold concentration of the quorum sensing signal generates a concerted response and (4) the cellular response extends beyond physiological changes required to metabolize or detoxify the molecule. When all four criteria are met, a molecule can be classified as QSM. The most interesting feature of these quorum sensing signals were well defined in the year 2002 by Winzer. These metabolites can induce, during their release, their own uptake system and the production of enzymes required for their breakdown. This indirectly influences the expression of genes from other linked metabolic pathways [7, 8]. Mathematical modelling is one of the important tools to study natural systems. Researchers like Anguige, Dockery, Ward and Majumdar have developed several mathematical models of quorum sensing systems based on experiments and analyzed the system in detail $[9,10,11,12,13,14,15,16]$. Still, certain important facts about QS systems are not understood properly. The hydrodynamics and the origin of noise in the QS mechanism are undiscovered. In this paper we sketch the dynamics of the QSM which has a velocity and we consider viscosity as ad hoc term $v \nabla^{2} \boldsymbol{u}$ which has an artificial effect in the fluid. We find out origin of noise in QS from the noisy Burgers equation and the internal structure of the quorum sensing mechanism using Zeldovich approximation which is analogous to pancakes [6]. In this present work weakly nonlocal fluid dynamical equation is utilized in which viscosity term is physically motivated.

\section{Origin of Noise and Internal Structure}

\section{Schrödinger-Madelung Fluid}

We consider that quorum sensing occurs in a Schrödinger-Madelung fluid with viscosity $v_{S c h M}$ and density $\rho$.The velocity of QSM is denoted by $\boldsymbol{v}$ which is given by the fluid. Let us define the peculiar velocity field as the vector filed $\mathbf{u}=\dot{\boldsymbol{r}}-H \boldsymbol{r}$ (where $H=\dot{a} / a$ and $a$ is a scale factor). The quantity $(\nabla \boldsymbol{u})_{\mathrm{ij}}$ is called the velocity gradient tensor, the symmetric part of this gradient is rate of strain tensor and antisymmetric part is the vorticity tensor [1].

Form Euler equation of peculiar velocity field we find 


$$
\frac{\partial \boldsymbol{u}}{\partial t}+\frac{1}{a}(\boldsymbol{u} \cdot \nabla) \boldsymbol{u}=-\frac{1}{a} \nabla \phi-\dot{a} / a \boldsymbol{u}+\frac{\mu}{\rho a^{2}} \nabla^{2} \boldsymbol{u}
$$

Where $\frac{\mu}{\rho}=v_{S c h M}$ is the kinematical viscosity, $\mu$ is known dynamical viscosity and changing the time scale from $t$ to $b$. After rescaling we get $\boldsymbol{v}=\frac{\boldsymbol{u}}{a \dot{b}}=\frac{d \boldsymbol{x}}{d b}, \mathrm{x}=\mathrm{r} / a(t)$ and

$$
\varphi=\left(\frac{3}{2} \Omega_{0} a^{2} b\right)^{-1} \phi
$$

Now using Schrödinger and Poisson equation and Hamilton - Jacobi canonical transformation we have,

$$
\begin{array}{r}
\frac{\partial v}{\partial b}+(\boldsymbol{v} . \nabla) v=\frac{3}{2 b} \nabla\left(\theta-\varphi-\phi_{v i s}\right) \\
\text { where } \phi_{v i s}=-\frac{v_{S c h M}^{2}}{2}\left[\frac{\nabla^{2} \rho}{\rho}-\frac{1}{2}\left(\frac{\nabla \rho}{\rho}\right)^{2}\right]
\end{array}
$$

For this weakly nonlinear $\operatorname{regime} \varphi \approx \theta$ and

$$
\frac{\partial v}{\partial b}+(\boldsymbol{v} \cdot \nabla) \boldsymbol{v}=-\frac{3}{2 b} \nabla \phi_{v i s}
$$

We define shear tensor as

$$
\sigma_{i j}^{k i n}=\frac{v_{S c h M}^{2}}{4}\left[\frac{\nabla_{i} \nabla_{j} \rho}{\rho}-\frac{\left(\nabla_{i} \rho\right)\left(\nabla_{j} \rho\right)}{\rho}\right]=\frac{v_{S c h M}^{2}}{4} \nabla_{i} \nabla_{j} \ln \rho
$$

We get $\nabla \sigma=-\nabla \phi_{\text {vis }}$

Now kinematical velocity is defined like

$$
u_{\text {kin }}=\frac{v_{S c h M}}{2} \nabla \ln \rho
$$

Finally from the symmetric part of the velocity gradient we have

$$
\frac{\partial v}{\partial b}+(\boldsymbol{v} . \nabla) \boldsymbol{v}=v_{S c h M} \nabla^{2} \boldsymbol{v}_{k i n}
$$

If we define $\Delta \boldsymbol{v}_{\text {kin }}=\boldsymbol{v}-\boldsymbol{v}_{\text {kin }}$, we write the general expression as

$$
\frac{\partial v}{\partial b}+(\boldsymbol{v} \cdot \nabla) \boldsymbol{v}=v_{S c h M} \nabla^{2} \boldsymbol{v}_{k i n}+\nabla \eta
$$

where $\nabla \eta=-v_{S c h M} \nabla^{2}\left\langle\Delta \boldsymbol{v}_{k i n}\right\rangle$ is a noise term .This equation is corresponds to noisy Burgers equation and Zeldovich approximation if $\boldsymbol{v}_{\text {kin }} \rightarrow 0$ which find out the importance of internal structure of quorum sensing.

\section{Alternative Fluid}

Now we change the fluid which has the nonlocal term in potential is $U_{A l t}=-\frac{v_{A l t}}{2} \Delta \rho$ where $v_{A l t}$ is consider as a Kinematical viscosity of this fluid [4].In this alternative fluid we find out $\phi_{v i s}=-\frac{v_{A l t}}{2} \nabla^{2} \rho$ and in the similar manner we obtain $u_{k i n}=\frac{1}{2} \nabla \rho$. Then from the symmetric part of the velocity gradient we write a general expression of the noisy Burgers equation as follows

$$
\frac{\partial v}{\partial b}+(\boldsymbol{v} \cdot \nabla) \boldsymbol{v}=v_{A l t} \nabla^{2} \boldsymbol{v}_{k i n}+\nabla \eta
$$

\section{Discussion}

This is a framework of the internal structure of the quorum sensing mechanism in bacteria .The source of internal noise in quorum sensing is originated from some perturbation due to velocity of the QSM. The kinematical quasivelocity $u_{\text {kin }}=\frac{v_{S c h M}}{2} \nabla \ln \rho$ and $u_{\text {kin }}=\frac{1}{2} \nabla \rho$ does not represent actual mechanical motion, but does contribute to the transfer of momentum and concentration of energy [5]. $\phi_{v i s}$ of quantum mechanics represent the probability distribution of the single QSM. The origin of viscosity term is also investigated in QS mechanism and this viscosity property which makes QSM together into caustics predicted by Zeldovich approximation [1]. Viscosity is also related to temperature. So QS in also connected to temperature of the fluid. Kinematical Viscosity $v_{S c h M}=\frac{\mu}{\rho}$ and $v_{\mathrm{Alt}}=\frac{\mu}{\rho}$ is referred to as diffusivity of momentum because it is analogous to diffusivity of mass. Quorum sensing mechanism depends on the internal noise. For this certain noise quorum sensing occurs and this communication process also can be destroy for a particular threshold of noise .Whenever the QSM is released by a single bacterium in the system fluid, which is assumed to follow Schrödinger-Madelung or Alternative fluid characteristic, it creates a noise. The event of the release of the quorum sensing molecule creates a source of fluctuation in the fluid which is carried out by the diffusion mechanism throughout the fluid .The possibilities of interaction with other bacterial cells increase with the inversing fluctuation made by the QSM .When the noise reached a threshold level the stationary takes place and this fluctuations is actually turned as quorum sensing .Consequently the group behavioural pattern changes by the manipulation of given expression.

\section{Conclusions}

This biochemical phenomenon is theoretically investigated here. For future it will be verified in experimentally. Using different fluid this quorum sensing mechanism and the dynamics of QSM can be investigated.

\section{REFERENCES}

[1] A.L.B. Ribeiro and J.G. Pexioto de Faria. Weakly nonlocal hydrodynamics and the origin of viscosity in the adhesion model, Physical Review D 71,067302 (2005).

[2] Fuqua, C., S. C. Winans and E. P. Greenberg Census and concensus in bacterial ecosystems: The luxR-luxI family of quorum-sensing transcriptional regulators. Annu. Rev. Microbiol. 50, 727-751. (1996).

[3] Miller MB, Bassler BL, Quorum sensing in bacteria. Annu Rev Microbiol, 55(2001) pp. 165-199.

[4] P.Van and T.Fulop, Weakly nonlocal fluid mechanics- The Schrödinger equation, arXiv: quant-ph/0304062 9 Jun 2004.

[5] R.J. Harvey, Navier-Stokes Analog of Quantum Mechanics, Physical Review, Vol 152 No 4, (1966). 
[6] Y.B.Zeldovich, Astron. Astrophys. 5, 84(1970).

[7] Winzer, Klaus, et al. "LuxS: its role in central metabolism and the in vitro synthesis of 4-hydroxy-5-methyl-3 (2H)-furanone.” Microbiology 148.4 (2002): 909-922.

[8] Winzer, Klaus, Kim R. Hardie, and Paul Williams. "Bacterial cell-to-cell communication: sorry, can't talk now-gone to lunch!." Current opinion in microbiology 5.2 (2002): 216-222.

[9] J.P.Ward, J.R. King, A.J. Koerber, P. Williams, J.M. Croft, and R.E. Sockett, Mathematical modeling of quorum sensing in bacteria, IMA Journal of Mathematics Applied in Medicine and Biology 18 (2001), pp. 263-292.

[10] Anguige K, King JR, Ward JP, Williams P: Mathematical modelling of therapies targeted at bacterial quorum sensing. Mathematical Biosciences, 192:39-83. (2004)

[11] Anguige K, King JR, Ward JP: A multi-phase mathematical model of quorum sensing in a maturing Pseudomonas aeruginosa biofilm. Mathematical Biosciences,
203(2):240-276. (2006)

[12] Dockery JD and JP Keener. A mathematical model for quorum sensing in Pseudomonas aeruginosa. Bull Math Biol 63: 95- 116. (2001).

[13] Sarangam Majumdar, Suman Datta, Sisir Roy, Mathematical modeling of quorum sensing and bioluminescence in bacteria, International Journal of Advances in Applied Sciences (IJAAS), Vol 1, No 3 pp.139-146 (2012).

[14] Majumdar, Sarangam. "Hydrodynamic Conditions of Quorum Sensing in Bacteria." International Journal of Biochemistry and Biophysics 1.1 (2013): 22-26.

[15] Mondal, Subhoshmita, and Sarangam Majumdar. "Mathemat ical Modelling of Beauveria Bassiana." Universal Journal of Applied Mathematics 2.1 (2014): 1-4.

[16] Majumdar, Sarangam, and Subhoshmita Mondal. "Perspecti ves on Quorum sensing in Fungi." International Journal of Modern Biology and Medicine", vol 6, No 3 pp. 170-180, (2015). 